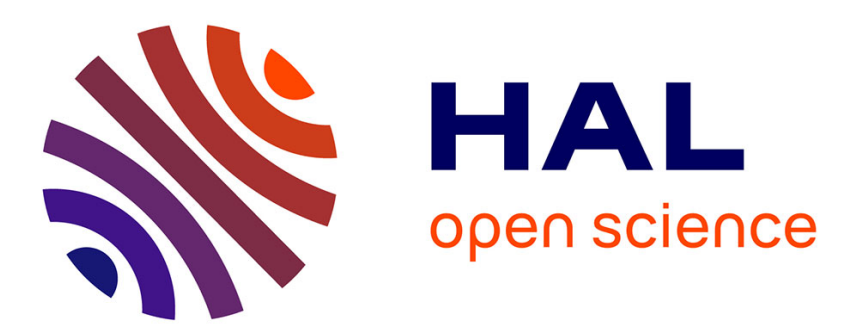

\title{
Modelling of hot cracking in welding with a cellular automaton combined with an intergranular fluid flow model
}

\author{
Cyril Bordreuil, Aurélie Niel
}

\section{To cite this version:}

Cyril Bordreuil, Aurélie Niel. Modelling of hot cracking in welding with a cellular automaton combined with an intergranular fluid flow model. Computational Materials Science, 2014, 82, pp.442 - 450. 10.1016/j.commatsci.2013.09.067 . hal-01444451

\section{HAL Id: hal-01444451 \\ https://hal.science/hal-01444451}

Submitted on 24 Jan 2017

HAL is a multi-disciplinary open access archive for the deposit and dissemination of scientific research documents, whether they are published or not. The documents may come from teaching and research institutions in France or abroad, or from public or private research centers.
L'archive ouverte pluridisciplinaire HAL, est destinée au dépôt et à la diffusion de documents scientifiques de niveau recherche, publiés ou non, émanant des établissements d'enseignement et de recherche français ou étrangers, des laboratoires publics ou privés. 


\title{
Modelling of hot cracking in welding with a cellular automaton combined with an intergranular fluid flow model
}

\author{
C.Bordreuil, A.Niel \\ Laboratoire de Mécanique et Génie Civil-UMR5508,CC048, Place Eugène Bataillon, Université \\ Montpellier 2, 34095 Montpellier, France
}

\begin{abstract}
Hot cracking phenomena in welds are still a major issue to choose suitable welding conditions. Solidification cracking in welds depends on microstructure morphology, thermal and mechanical loadings. It is still difficult to discriminate the influence of the different phenomena because they are all connected. To investigate solidification cracking, numerical modelling that integrates most of these phenomena is developed in this paper. Thermal and mechanical fields are computed with a finite element model at the process scale. The thermal field is combined with a cellular automata (CA) to predict microstructure at a meso scale. Based, on this prediction, the intergranular network is extracted. Finally, the pressure in the intergranular fluid network is computed during solidification. The fluid flow model along grain boundaries predicts an eventual nucleation of pore/void in the remaining liquid by investigating the maximum pressure drop in the solidifying microstructure. The numerical model is able to describe some hot cracking phenomena observed during experimental tests.

Keywords: Hot cracking, Welding, Microstructure Prediction, Grain boundaries, Fluid network, Solidification
\end{abstract}

\section{Introduction}

Ensuring crystal cohesion during solidification in welding by avoiding cracks is a major challenge in material science and engineering. Factors affecting weld metal solidification cracking can be considered as either metallurgical or mechanical and often

Email address: cyril.bordreuil@univ-montp2.fr (C.Bordreuil, A.Niel) 
interact together. The weld solidification cracking of alloys often appears in the mushy zone. This zone is a transition region between the weld pool and the base material. The behaviour of this region is complex because it mixes liquid and solid phases that interact under solidification. Different regions depending on the solid fraction, can be defined at the microstructure scale [1]. The first region correspond to a high liquid fraction around dendrites where liquid can easily flow around solid skeleton. The second region is for high solid fraction but still with a continuous liquid network around dendrites or along grain boundaries. The last region is at a very high solid fraction with isolated liquid films and solid bridges are created between grains. In general, the coherency solid fraction $f_{\text {coh }}$ is defined around the transition between the two last regions. It is known from casting that defects as hot tearing and porosities can be due to solidification shrinkage and/or to tensile strain rate. Solidification shrinkage and plastic strain rate opening has to be compensated by fluid flow. If less flow is available, a depression can be created and a pore can nucleate. One important point that is rarely taken into account is the morphology of the intergranular fluid network and the flow along it. By using a microstructure prediction, the model is able to take into account this feeding along grain boundaries.

In welding, the hot cracking phenomenon is difficult to predict due to high gradient arising in the vicinity of the weld pool. These gradients load the solidifying microstructure. In particular, tensile stresses can be generated by differential thermal shrinkage upon cooling or by difference of plastic behaviour [2]. It is also often emphasized that grain morphology has an influence on hot cracking [3]. Due to fast cooling rate induced by the process, the microstructure morphology is heterogeneous at micro, meso and macroscale. At the microscopic scale, the grain demonstrates a dendritic substructure [4]. At the weld bead scale, the process generates a morphology with columnar and equiaxed grains. There is a transition from a columnar to equiaxed dendritic morphology called CET (columnar equiaxed transition). For the $6061 \mathrm{Al}$ Alloy, the crack onset was observed in the columnar zone, far from the the weld pool in region of high solid fraction [5] and at grain boundaries. The solidification shrinkage and/or tensile stresses can create a depression in the interdendritic spacing or at grain boundaries. Tensile stresses perpendicular to the grain boundaries tend to create an opening at grain boundaries.

The literature on hot cracking phenomena in welding is abundant $[6,7,8]$. Chihoski 
[2] demonstrates, first, the influence of structural effects induced by the localized heat input. Matsuda [9] introduced the Brittle Temperature Range (BTR) based on observation. The BTR defined a temperature interval where the microstructure is in a critical configuration. In this model, strain rate and temperature are used to predict crack onset and doesn't introduce any metallurgical considerations (or variables). On superalloy, Wang [3] adapted a criterion of Rappaz [10] for hot tearing to the case of hot cracking in welding of single and bi crystals. The Rappaz - Drezet - Gremaud [10] (noted RDG in the rest of the text) criterion introduced some microstructural variables as well as macroscopic variables. This model can take into account the influence of lack of liquid feeding as well as some plastic strain localisation in the formulation. The variables are linked with the theory of multiphasic media and was able to predict crack onset in some configuration of welding [3]. Because, welding induces characteristic length (grain size, weld pool width) of the same order of magnitude, hot cracking susceptibility modelling needs an integrated approach to take into account process, microstructural morphology and liquid-solid phases under solidification. Due to the difficulty to measure mechanical and thermal fields in the vicinity of the moving weld pool, computational modelling must be performed to approximate stress/strain distribution in the mushy zone and to understand the influence of the different mechanisms on the hot cracking [11]. The modelling needs to integrate process simulation, microstructural prediction and depression computation in the intergranular fluid network.

The purpose of this paper is to propose a methodology to investigate the couplings that can coexist between process and microstructure. It describes the modelling to approximate pressure drop in the continuous intergranular network. The computational model that combines thermo-mechanical, cellular automaton and pressure computation during the solidification is explained. The process simulation is done with a finite element thermo-mechanical model. The microstructural prediction is based on a cellular automaton based on solidification mechanisms driven by the thermal field. The pressure in the liquid network is then computed by adapting the RDG model to the microstructure predicted by the cellular automaton algorithm and loaded with thermal and mechanical fields. Some comments are also given on the influence of the coalescence of grain boundaries. Then, some results given by this approach are shown. The methodology 
is depicted with some two dimensional simulations done on 6061 Aluminium Alloy that was compared with some experiments [5]. The relationship between microstructural prediction and fluid flow at grain boundaries is new an try to contribute to the explanation of hot cracking in welding.

\section{Computational model}

Different phenomena, acting at different scales, are involved in weld solidification. To model this phenomenon, different scales must be integrated:

- process scale : thermal and mechanical fields (strain rate).

- microstructure : fluid pressure computation around the solid skeleton in the intergranular fluid network.

To determine the temperature and stress/strain fields in the mushy zone, a thermomechanical finite element model is performed. This model allows to estimate fields during welding process. These fields are determined at the (macroscopic) process scale and drive the microstructure generation. To predict cracking, the microstructure morphology has to be determined. A cellular automaton (CA) is implemented to directly track grain morphology. The CA can take into account different physical mechanisms as competitive grain growth, grain selection and CET. In welding, the microstructure is assumed to be driven by the process and the coupling between microstructure evolution and thermal field can be considered as weak [12]. In this model, micro and macro segregation are not taken into account. The CA results give grains boundaries but also crystallographic orientation and solid fraction can be estimated with thermal fields. Once microstructure is known, the pressure at grain boundaries can be determined by adapting the finite element RDG model developed in [5].

The integrated algortihm considering different modelling is organized as follows:

1. Thermo-mechanical finite element model of the process to determine macroscopic fields.

2. Microstructural prediction with CA.

3. Conversion of CA data structure to multiphasic finite element to predict pressure in the intergranular network. 
Then by comparing the pressure the intergranular network to cavitation pressure in as in RDG criterion [10], the crack susceptibility can be determined. In this model, the important effect of hydrogen content [13] can be taken into account by a dependence of the cavitation pressure to the hydrogen content as explained in [14].

In the next sections, the different modeling are briefly discussed.

\subsection{Thermo-mechanical finite element model}

The model is conducted to simulate the behaviour of thin plate for full penetrated weld pool. For the model under consideration, it is not necessary to solve temperature and stress/strain fields simultaneously due to the weak couplings between the two problems. An uncoupled thermal and thermo-mechanical analysis is adopted. The thermal transient analysis is first performed followed by a quasi static mechanical analysis loaded by the thermal history. In this model, the thermal analysis is solved with an enthalpy formulation and no convection is taken into account for simplicity. Nevertheless, the strategy could be adapted if an analysis similar to Farzadi et al [15] is performed by using a thermo convective model to compute thermal fields.

\subsubsection{Heat transfer model}

A two dimensional heat plane analysis is performed. The dimension of the plate is 400x100x3 mm. The material is 6061 Aluminium Alloy. Only half of the plate is discretized due to the assumption of symetry along the centre line. Elements are quadrangle isoparametric elements in the middle and outer part of the plate. A transition region is meshed with triangular elements. The arc heat input to the workpiece is modelled as a flux with a gaussian distribution with $\mathrm{r}$ the distance from the arc center:

$$
q(r)=q_{\text {max }} \exp \left(-3\left(\frac{\sqrt{\left(x-x_{0}\right)^{2}+\left(y-y_{0}-V t\right)^{2}}}{r_{0}}\right)^{2}\right)
$$

The $q_{\max }=\frac{\eta U I}{3 \sqrt{\pi} r_{0}^{2}}$ is a surface flux $\left(W / m^{2}\right)$ related to process parameters arc voltage $\mathrm{U}$ and current $\mathrm{I}, \mathrm{V}$ the welding speed and where $\eta$ is the efficiency of the process and $r_{0}$ characterized the radius of the heat distribution.

In the heat transfer, heat is lost from the surface in the form of convection and radiation on all surfaces. The thermal model is conducted considering an enthalpy formulation considering latent heat release in the solidification range of the alloy. The enthalpy is 


\begin{tabular}{|c|c|}
\hline Liquid Density $\left(\mathrm{kg} / \mathrm{m}^{3}\right)$ & 2200 \\
\hline Solid Density $\left(\mathrm{kg} / \mathrm{m}^{3}\right)$ & 2700 \\
\hline Capacity $(\mathrm{J} / \mathrm{kg} / \mathrm{K})$ & 896 \\
\hline Conductivity $(\mathrm{W} / \mathrm{K} / \mathrm{m})$ & 200 \\
\hline Volumetric Latent Heat $\mathrm{J} / \mathrm{m}^{3}$ & $10^{9}$ \\
\hline Exchange coeffcient $\mathrm{W} / \mathrm{m}^{2} \mathrm{~K}$ & 10. \\
\hline
\end{tabular}

Table 1: Parameters used in the thermal transient analysis

computed with the integration of the solid fraction in the solidification interval. Because the coupling between process and metallurgy wants to be analysed, the solid fraction is considered not dependent of the solidification path in the whole domain. This approximation is strong but ease the analysis of the coupling (see section 4). The enthalpy is tabulated with respect to the temperature. All the parameters are summarized in table 1. More details on the thermal transient analysis can be found in $[5,16]$.

\subsubsection{Mechanical model}

The geometry and the mesh are the same as the thermal analysis. To estimate the mechanical fields, the important ingredients are:

- The mechanical behaviour of the material.

- The deformation in the molten weld pool.

- The solidification shrinkage.

First, the mechanical behaviour of the 6061 alloy was deeply investigated under a wide range of temperature by Maisonnette [17]. A mixed kinematic-hardening model was chosen and restauration is taken into account with two internal variables representing the state of the microstructure. One variable represents the initial microstructure and the other represents the microstructure with dissolved precipitates. In the mushy zone, the volumetric plastic strain rate [18] is not taken into account. It is questionable but it seems that structural effects plays a more important role on the plastic strain state in the mushy zone than its behaviour.

Secondly, the weld pool can hardly sustain mechanical load which can be modelled as a stress free region. Previous plastic strain of the resolidified weld metal has to be 


\begin{tabular}{|c|c|c|}
\hline Property & Ambient & $500 \circ C$ \\
\hline Young Modulus (MPa) & 70000 & 38000 \\
\hline Poisson ratio & 0.3 & 0.3 \\
\hline Yield Limit (MPa) & 280 & 80 \\
\hline Dilatation coefficient (/K) & $27 \mathrm{e}-6$ & $35 \mathrm{e}-6$ \\
\hline
\end{tabular}

Table 2: Parameters used in the mechanical analysis

annealed. These two phenomena are modelled by defining a coherent temperature above which stresses and plastic strains are set to zero.

Third, the solidification shrinkage has a significant impact on stresses and strains. Because, thermal stresses of an alloy is concerned, the effect of solidification shrinkage is modelled as thermal contraction in solidification temperature range. Some mechanical parameters are summarized in table 2 .

More details on the mechanical models can be found in [16].

\subsubsection{Thermal and mechanical fields}

When the analysis are finished, the fields are extracted from the results databases. The temperature are known at nodes. Thermal fluxes and plastic strains are extrapolated from gauss points to nodes. All fields are kept at nodes with respect to time. Then, these fields could be interpolated to any subdomain. The method to interpolate these values on the microstructural domain $(\mathrm{CA})$ is a natural neighbour interpolation [19]. This interpolation has the advantage to let the procedure find the nearest points to build the shape functions.

\subsection{Cellular Automaton}

The cellular automaton has been already used to predict microstructure during welding process in two dimension $[12,20]$ and in three dimensions [21]. The temperature at microstructural scale is directly governed by the thermal transfer at the process scale. In welding process, the thermal solution can be uncoupled for the microstructural prediction. To investigate the interest to study an integrated modelling with microstructure prediction and process simulation, a two dimensional Cellular Automaton was implemented. The two dimensional thermal field coming from the process modelling is used to feed modelling of physical mechanisms present during solidification. The main physical phenomena are : 
- Nucleation of new grain in the liquid or on a wetting grain.

- Growth of dendritic structure from existing grains or newly nucleated ones.

- Coalescence of remaining liquid films or isolated pocket.

The CA algorithm follows the development of $[12,22]$. Some important features are reminded. The CA domain is defined as a structured grid of cells. Each cell $\nu$ has a state index $I^{\nu}$ and a crystallographic index $I^{C}$. The state index allows to know if the cell is melt, partially melt or solid. The CA domain is chosen in a part of the macroscopic domain where the crack is assumed to occur. A reduced domain allows to accelerate computational to appreciate the interest of these kinds of modelling. The CA domain is initialized by defining a voronoi diagram on the domain. The orientation (an angle in 2D) of each voronoi cell is assigned randomly. The voronoi cell size is chosen in order to match the grain size of the base material. If CA cell center belongs to one voronoi cell, the crystallographic orientation of the voronoi cell is assigned to the CA cell.

To determine CA cell that has melt, the temperature field is used. CA cell with its center at a temperature higher than the melting temperature has its state index set to MELT. Cell in the border of the weld pool has their state index set to PARTIALLY_MELT. In $2 \mathrm{D}$, the growing shape of a cell is a square with the diagonals aligned with the crystallographic direction. One of the main key point of CA is the propagation of the shape. The neighbour cell is captured when the parent cell has its shape that reaches the cell centre. The neighbouring cell in MELT state has its state index assigns to PARTIALLY_MELT and the crystallographic orientation is set to the parent one. A new smaller shape is associated with the capture cell. The growth is stopped if a neighbouring cell is in a PARTIALLY_MELT state. The shape grows according to speed of growth $v_{g}$ which depend on undercooling and species concentration.

Thermal fields are known at times $t_{n}^{\text {proc }}, t_{n+1}^{\text {proc }}$ where $n$ subscript stands for the time step of the process simulation. The process and metallurgical modelling haven't got the same characteristic times and must be synchronized. The time increment at the process scale is denoted $\Delta t$. The $\mathrm{CA}$ algorithm time increment $\delta t$ has to be limited in order to have effective prediction [22]. Times for CA are denoted: $t_{k}^{C A}, t_{k+1}^{C A}$. Fields needs to be interpolated from the process domain to the $\mathrm{CA}$ domain with the different time steps 
(figure 1).

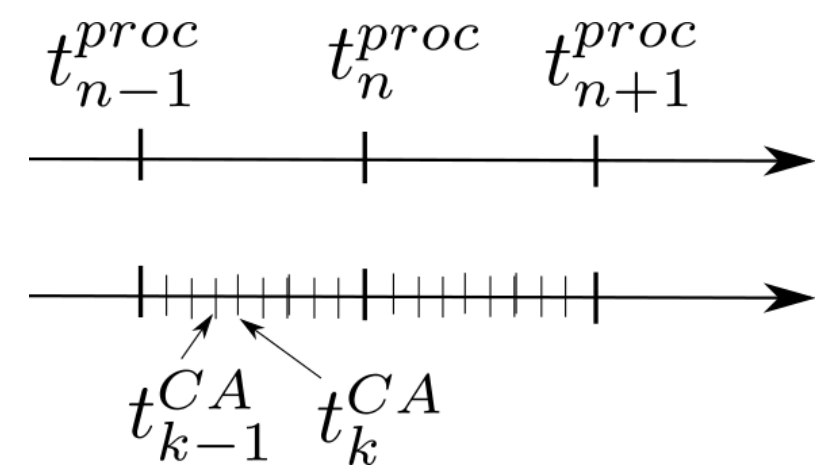

Figure 1: Synchronization of the different time steps used in the coupled model.

To know temperature for each time step of the CA algorithm, a temporal interpolation is performed between times $t_{n-1}^{\text {proc }}, t_{n}^{\text {proc }}$ and $t_{n+1}^{\text {proc }}$ using a parabolic scheme. The temperature is kept in a First In First Out queue.

$$
T^{C A}\left(t^{C A}\right)=a\left(t^{C A}\right)^{2}+b t^{C A}+c
$$

where a,b,c are constant. They are determined with the condition: $T^{C A}\left(t_{i}^{\text {proc }}\right)=$ $T^{\text {proc }}\left(t_{i}^{\text {proc }}\right)$ with the three temperatures in the FIFO queue.

Then, the microstructure is predicted following the CA algorithm depicted on table 3 and reimplemented from $[12,22]$.

1. For each process time step $t_{n}^{\text {proc }}$

(a) Assign new temperature in the FIFO queue.

(b) Interpolate temperature for current time step

(c) Find CA cells up to liquidus temperature and assign $I^{\nu}$ to MELT.

(d) For each CA time step

i. For each cell in PARTIALLY_MELT state compute the dendritic growth with the undercooling $\Delta T$

ii. For each cell in MELT state compute the nucleation with undercooling $\Delta T$

(e) if $t^{C A}=t^{\text {proc }}$ jump to next process step.

Table 3: CA algorithm used to predict the microstructure

The microstructure morphology is driven by several mechanisms that are described in welding by Kou [23]. 
Nucleation To take into account the nucleation of grains below the liquid temperature of the alloy, an athermal model is used [1]:

$$
\frac{d n}{d \Delta T}=\frac{n_{\max }}{\Delta T_{\sigma} \sqrt{2 \pi}} \frac{1}{\Delta T} \exp \left(-\frac{1}{2}\left(\frac{\ln \Delta T-\ln \Delta T_{0}}{\Delta T_{\sigma}}\right)^{2}\right)
$$

where $n$ is the grain density, $\Delta T$ the total undercooling, $\Delta T_{\sigma}$ is the standard deviation and $\Delta T_{0}$ is the mean undercooling.

Growth The growth takes place in this direction and the growth speed followed a Kurz, Giovanola et Trivedi model[24]:

$$
v_{g}=A \Delta T^{n}
$$

where $\mathrm{A}$ and $\mathrm{n}$ depends on depend on species concentrations in the alloy. This law was identified from Ivantsov solution and can be identified for binary alloys. Recently, it was shown that this law can be adapted to multicomponent alloy [25] by introducing local linearized multicomponent phase diagram. Normally, microsegregation in the intergranular fluid network needs to be integrated in the modelling. Difference of concentrations would lead to difference in $v_{g}$.

Coalescence Rappaz et al [26] introduced a model to take into account the fact that bridging of dendrites occurs later at grain boundaries for two grains with different crystalographic orientations. This model was used in welding process of single crystals by Wang et al [3] to investigate hot cracking. A bridging undercooling is defined for a pure metal as:

$$
\Delta T_{b}=\frac{T_{m}}{L_{f}} \frac{\gamma_{g b}-2 \gamma_{s l}}{\delta}
$$

where $\gamma_{g b}$ is the grain boundary surface energy, $\gamma_{s l}$ the solid liquid surface energy, $L_{f}$ the latent heat of fusion and $T_{m}$ the temperature of fusion and $\delta$ is the thickness of the remaining liguid and is of the order of $\mathrm{nm}$. The grain boundary surface energy is given by the Read-Shockley law $[3,26]$.

\subsection{RDG Finite Element Model}

The grain substructure is mainly dendritic due to fast cooling rate. A typical weld microstructure is depicted in figure 2 . 


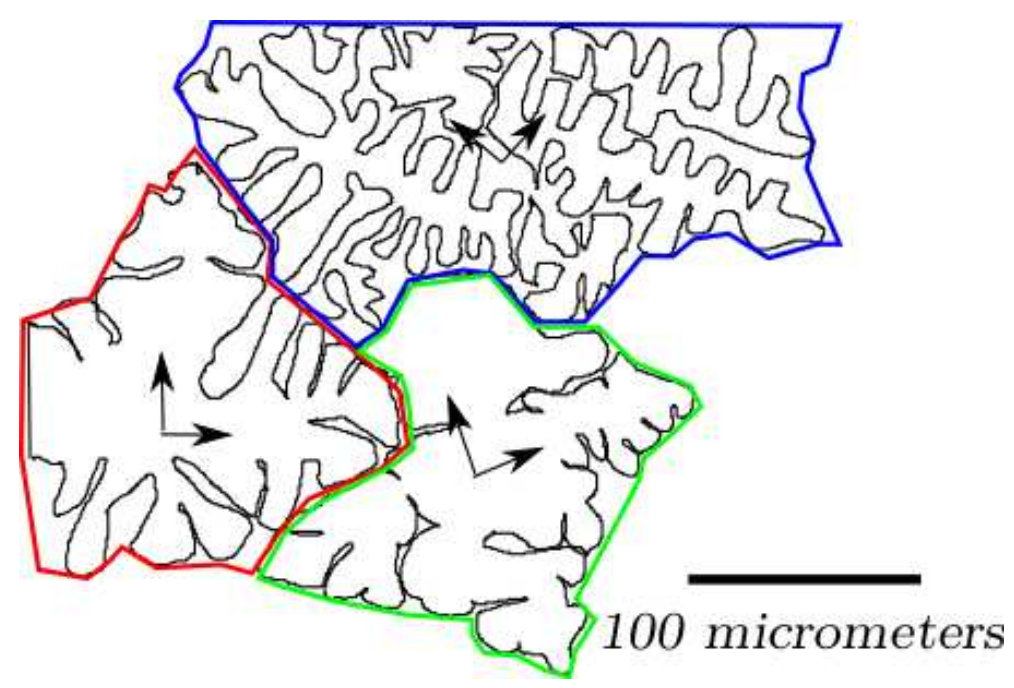

Figure 2: Dendritic structures configuration after welding of 6061 Aluminium alloy in a equiaxed zone

On figure 2, the main characteristics of the microstructure are shown:

- Primary arm-dendrite spacing

- Secondary arm-dendrite spacing

- Crystallographic orientation

- Grain boundaries

Because hot cracking seems to happen around the coherent solid fraction, the fluid flow along grain boundaries want to be modelled until this solid fraction $f_{c o h}$ is reached. To model fluid flow along grain boundaries, several models can be developped. The first one is to reproduce the development of Vernède [27] for globular grains. This modelling considers Poiseuille flow and mass exchange under solidification. This model seems to be the right one for flow around grain under solidification. For our study, the fluid flow has to be modelled in the last stage of solidification near $f_{\text {coh }}$. At this solid fraction, the grain boundary is tortuous due to the meeting of dendrites. The grain starts to sustain mechanical loads and strain localization can occurs along grain boundaries. Even if RDG model is adapted to regular arrangement of dendrites, it can be interesting to apply it to grain boundaries. 
The RDG model was adapted [5] to take into account heterogenous fields. This model is then considered to model fluid flow along grain boundaries. The weak formulation is reminded here.

$$
\int_{L} \mathbf{B}^{T} \mathbf{K B} d x\{p\}=\int_{L} \mathbf{N}^{T}\left(\beta \frac{d f_{s}}{d T} \dot{T}+(1+\beta) f_{s} \dot{\varepsilon}_{p \perp}\right) d x
$$

$\mathbf{N}$ is a shape function and $\mathbf{B}$ is the derivative of the shape function and $\{p\}$ is the vector of degree of freedom. Where $\mathrm{L}$ is the length of a grain boundary element, $\mathrm{K}$ its permeability, $f_{s}$ is the solid fraction, $\dot{\varepsilon}_{p \perp}$ is the plastic strain rate perpendicular to the grain boundary. As given in [28], the plastic strain rate is given by equation 7 .

$$
\dot{\varepsilon}_{p \perp}=K_{\text {localization }} \dot{\bar{\varepsilon}}_{p \perp}
$$

where $\dot{\bar{\varepsilon}}_{p \perp}$ is the average plastic strain rate deduced from the continuous mechanical model and $K_{\text {localization }}$ is a localization factor. This factor can depend on several microscopic factors. This value was approximated to $\phi / \delta$ where $\phi$ is the grain size and $\delta$ is proportional to the width of the grain boundary. At the coherent solid fraction, this length is around several nanometers. The grain is measured around $100 \mu \mathrm{m}$. The average plastic strain rate in plane stresses (in plane with axis 1,2) is computed with plastic strain rate at quadrature points. The perpendicular strain rate is calculated according to equation 8 .

$$
\dot{\bar{\varepsilon}}_{p \perp}=\cos ^{2}(\theta) \dot{\bar{\varepsilon}}_{11}^{p}+\sin ^{2}(\theta) \dot{\bar{\varepsilon}}_{22}^{p}+2 \cos (\theta) \sin (\theta) \dot{\bar{\varepsilon}}_{12}^{p}+\dot{\bar{\varepsilon}}_{33}^{p}
$$

In the modelling $\dot{\bar{\varepsilon}}_{p \perp}$ is taken into account if it is positive and negative. Negative values has a benefit effect if solidification shrinkage is important. $\dot{\bar{\varepsilon}}_{33}^{p}$ is taken into account because if tensile strain rate is observed it has to be compensated by liquid feeding. $\theta$ is the orientation of the grain boundary in the macroscopic frame.

The model is able to predict pressure drop due to solidification shrinkage as well as strain localisation with the plastic strain perpendicular to the grain boundary. The right hand term of equation 6 correspond to source terms. The integration is performed by a gauss quadrature along line element representing the grain boundaries. The internal variables in the source terms (right hand side of equation 6) are interpolated from the thermal and mechanical process simulation (see section 2.1.3). 
One of the important point of the model is the solid fraction evolution vs temperature. In the model, the 6061 solid fraction evolution is taken from [29] where it was computed with the help thermo-calc database. The solid fraction of 6061 Alloy is then tabulated in the solidification range defined from 540 to $645^{\circ} \mathrm{C}$. The solid fraction was chosen only dependent on the temperature. In reality the solid fraction evolution will depend on segration occuring at micro scale. Microsegregation can be different from point to point. Before refining the model by taking into account this phenomenon, this evolution of the solid fraction is chosen to be the same for the whole domain, to appreciate the reliability of the approach that couples microscale modelling and process simulation.

The other important ingredient is on the left hand side in the computation of the stiffness matrix with the approximation of the permeability. In such computation, the Carman-Kozeny is normally used to predict flow (equation 9).

$$
K=\frac{\lambda_{2}^{2}}{180} \frac{\left(1-f_{s}\right)^{3}}{f_{s}^{2}}
$$

where $\lambda_{2}$ is the spacing in the direction perpendicular to the fluid flow. Here, it corresponds to the final width of primary arm-spacing and it was estimated to $10 \mu \mathrm{m}$ with micrographies. Equation 9 is the permeability for two dimensionnal flow. In the case of solidifying microstructure, the grains are not two dimensionnal and the fluid can come from the third direction and ease the fluid flow. The permeability is often higher for a three dimensional microstructure.

\subsection{Conversion from $C A$ domain to RDG Finite elements}

The RDG based on Darcy and mass-conservation equations must normally be solved in the mushy zone. Grain boundaries are detected by scanning all CA cell and by looking edges along which $I^{C}$ changes from cell to cell. The edge is kept in a list. This list will contains all segments that will be converted in one dimensional RDG finite element model [5]. Not all edges are converted to a RDG Finite element model. To insert edges in the model, the solid fraction at the midpoint is computed. If the solid fraction is less than the coherent solid fraction, the edges is inserted in the model. At the end a continuous network is obtained where the different stiffness and right hand side can be assembled to obtain the system of equations on the whole domain: 


$$
\mathbf{K}\{\mathbf{p}\}=\mathbf{F}
$$

where $\{\mathbf{p}\}$ is the pressure vectors on the whole microstructure .

The system of equations is augmented by a set of boundary conditions. In this work, it is assumed that when the solid fraction is lower than 0.5 the pressure is imposed to the atmospheric pressure. Once the pressure is known along grain boundaries, the location with the highest pressure drop in the solidification interval can be determined.

Each segment of the grain boundaries assumed to have a continuous liquid films (under $\left.f_{c o h}\right)$ has a 'activated' tag. Grain boundaries along grains with different orientations have still liquid films at solid fraction higher than $f_{c o h}$. To take into account this fact, coherent solid fraction is modified to take into account the coalescence phenomena. A new coherent solid fraction is defined based on the coalescence undercooling. The coherent temperature is defined as the temperature corresponding to the coherent solid fraction. Then, the coalescence undercooling is substracted to finally obtain the new coherent solid fraction. Grain boundaries satisfying the criteria to have a solid fraction lower than the coherent solid fraction are not integrated in the model but has their tag 'activated' set to True (1). This can lead to isolated liquid films.

\section{Results}

The model is applied to an hot cracking test developed at the lab. The test consists in a simple autogeneous bead on 6061 Aluminium Alloy [5]. The welding speed is around $500 \mathrm{~mm} / \mathrm{min}$, the current is $200 \mathrm{~A}$ and the arc voltage is around $10 \mathrm{~V}$. Thermal and mechanical results are investigated and details in [5]. In [16], the thermal model was compared with thermocouples and weld bead size and gives good correlations. The overall configuration of the coupon is shown on figure 3. The first domain corresponds to the plate, it is the process domain. The second domain is the CA domain where all microstructure evolutions will be performed.

\subsection{Microstructural prediction}

In autogeneous TIG welding, the macroscopic grain structure is controlled by a combination of thermal conditions that prevails at the solid-liquid interface and crystal growth 


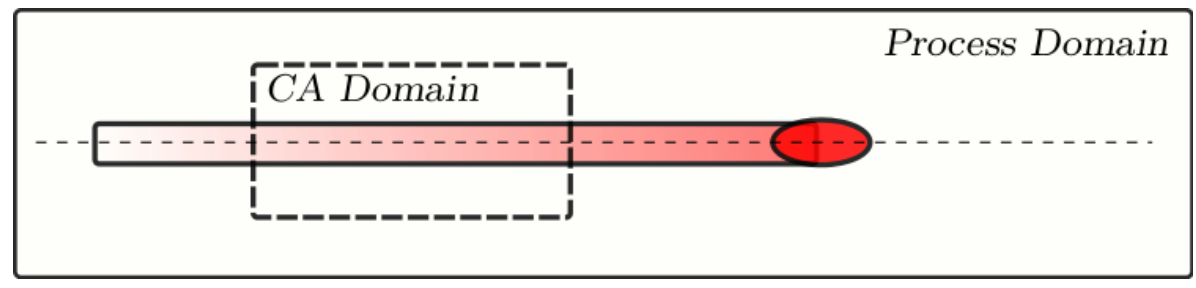

Figure 3: Domains induced in the modelling of the cracking test.

\begin{tabular}{|c|c|}
\hline $\mathrm{A}\left(\mathrm{m} / \mathrm{s} / \mathrm{K}^{n}\right)$ & 0.01 \\
\hline $\mathrm{n}$ & 2 \\
\hline$\Delta T_{\sigma}(\mathrm{K})$ & .5 \\
\hline$\Delta T_{0}(\mathrm{~K})$ & 5. \\
\hline$n_{\max } \mathrm{grain} / \mathrm{mm}^{3}$ & 1. \\
\hline$k$ & 0.05 \\
\hline$T_{m}\left({ }^{\circ} \mathrm{C}\right)$ & 645 \\
\hline$T_{s}\left({ }^{\circ} \mathrm{C}\right)$ & 540 \\
\hline$\mu\left(\mathrm{Pa.s}^{-} 1\right)$ & $1 . \mathrm{e}-3$ \\
\hline$f_{\text {coh }}$ & 0.92 and 0.96 \\
\hline
\end{tabular}

Table 4: Solidification parameters used in the microstructural prediction

rate which is directly related to welding speed. The conditions vary considerably depending on the position at the solid-liquid interface. At the trailing edge, solidification rate is high and favours formation of dendritic equiaxed grain that can be seen in the center of the weld pool. On either side of this central axial grains, columnar dendritic grains were found and grew towards the central axial region. The solidification of these grains is driven by the maximum thermal gradient or undercooling. The width of the equiaxed zone is around $4 \mathrm{~mm}$ and columnar zone is around $2 \mathrm{~mm}$.

Metallurgical parameters used are given in table 4.

In figure 4, the predicted microstructure for the welding test is shown. the model reproduces the competition growth from the base material and the nucleation mechanism at the trailing edge. The size of the equiaxed grains is more or less reproduced as well the orientation of the columnar grain. The model is able to reproduce the columnar to equiaxed transition observed during the test [5].

In figure 5, the black arrow is for the primary arm-dendrite direction in the columnar grain and the blue line is for the secondary arm-dendrite direction for the columnar grain. For the equiaxed grain, the two directions are equivalent. 

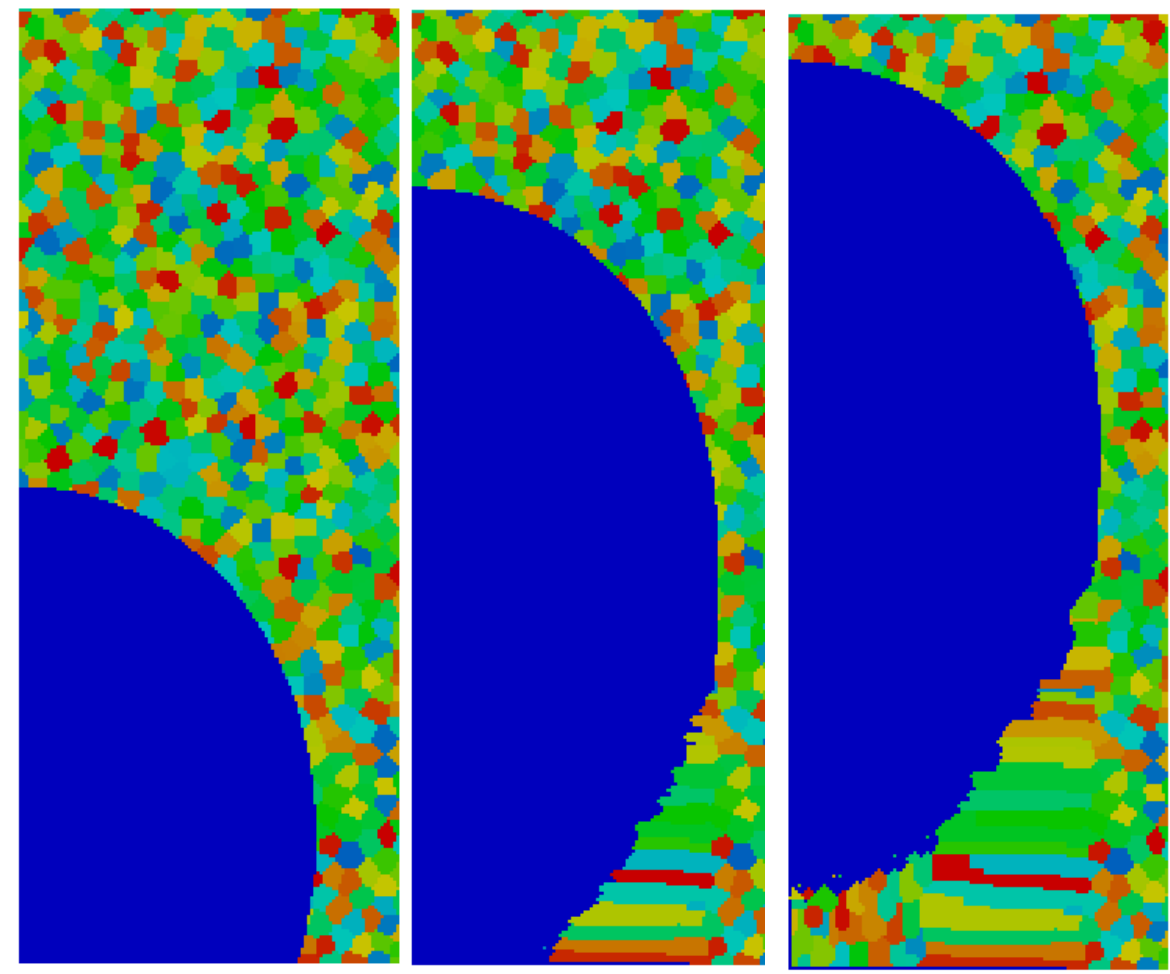

Figure 4: Evolution of the microstructure during moving arc heat input.

Time analysis of microstructure generation reveals that grains are nucleated in front of columnar grains due to a high undercooling and it activates the nucleation phenomenon. These grains have no parent grain and grows in the thermal field. The crystallographic orientation is assigned randomly and the grain type is set to equiaxed. There is then a competition with the growth of this grain and the growth of the columnar grain, explaining the columnar to equiaxed transition.

\subsection{Remaining liquid drops}

Remaining liquid films in tensile stresses region can be critical for hot tearing. A grain boundary element has its tag 'activated' when its solid fraction is lower that the coherency solid fraction. If grain boundaries have solid fraction higher than the coherency solid fraction it means that bridges was created between each grains leading to isolated liquid drops. With grain misorientation on each side of the grain boundary, a higher undercooling (bridging undercooling) is necessary to join both grain. This means that the 


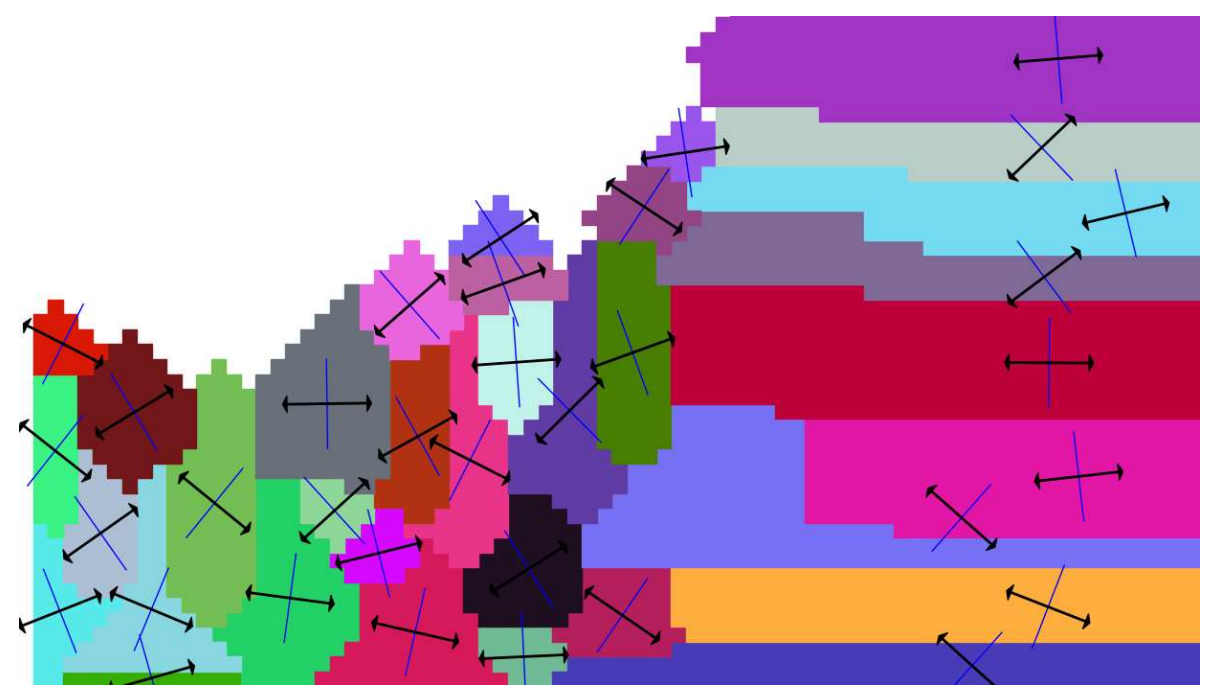

Figure 5: Two dimensionnal microstructural prediction. A grain is represented by a domain of same color. The microstructural observation reproduces Columnar to equiaxed transition (CET)

coherency solid fraction is increased for this part, liquid films stays at a lower temperature at grain boundary. In figure 6 , the 'activated' tag is plotted. If tag is equal to 1 , it means that the model predicted liquid films along grain boundaries mainly due to the coalescence undercooling.

In figure 6, the red large edges represented grain boundaries which have still remaining liquid films. Some grain boundaries are isolated from the intergranular fluid network. These films can not be feed by liquid coming from the weld pool. These isolated liquid films are critical sites for crack onset.

\subsection{Pressure drop}

In figure 7 , the result of pressure is obtained for the system of equations 10 with a coherent solid fraction of 0.92 .

In figure 7 , The highest pressure drop can be located and gives the critical site for crack onset. In this simulation, the highest pressure drop is in a grain in the last stage of solidification. This means that if pressure drop is sufficiently high to create a pore, a crack will certainly appears inside the columnar grain boundaries and it is located far from the weld pool. This indicates the importance of the microstructure and in particular the grain boundaries network to feed parts in the last stage of solidification with liquid. 


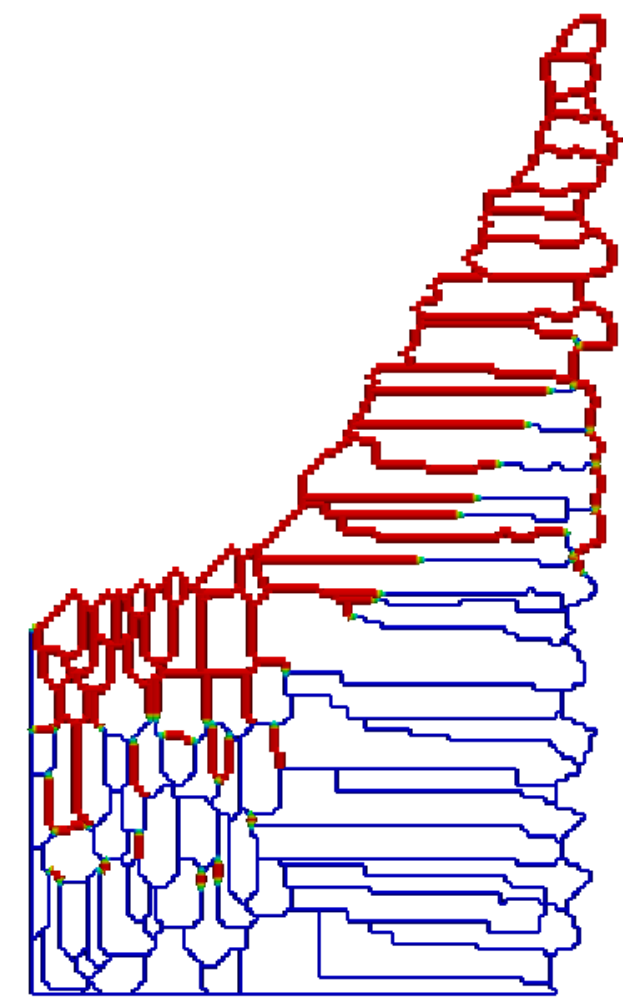

Figure 6: Activated tag along grain boundaries. Liquid grain boundaries are depicted in red color and with large segments.

The order of magnitude is $8 \mathrm{kPa}$ of depression. It is a little bit larger than those found in the litterature but it is of the same order of magnitude. This prediction is in accordance with some observations during GTAW of 6061 aluminum alloy [5].

\subsection{Coherency solid fraction influence}

The coherency solid fraction is not well defined. It can vary between 0.92 and 0.96 . If the pressure computation is performed with the second solid fraction, a higher depression will be found. It means that more liquid films coexist with solid, more the succeptibility of crack onset is important. The modification of the coherent solid fraction also modifies the grain boundary incorporation in the model. If the the coherent solid fraction goes from 0.92 to 0.96 , grain boundaries with lower permeability are introduced in the modelling 


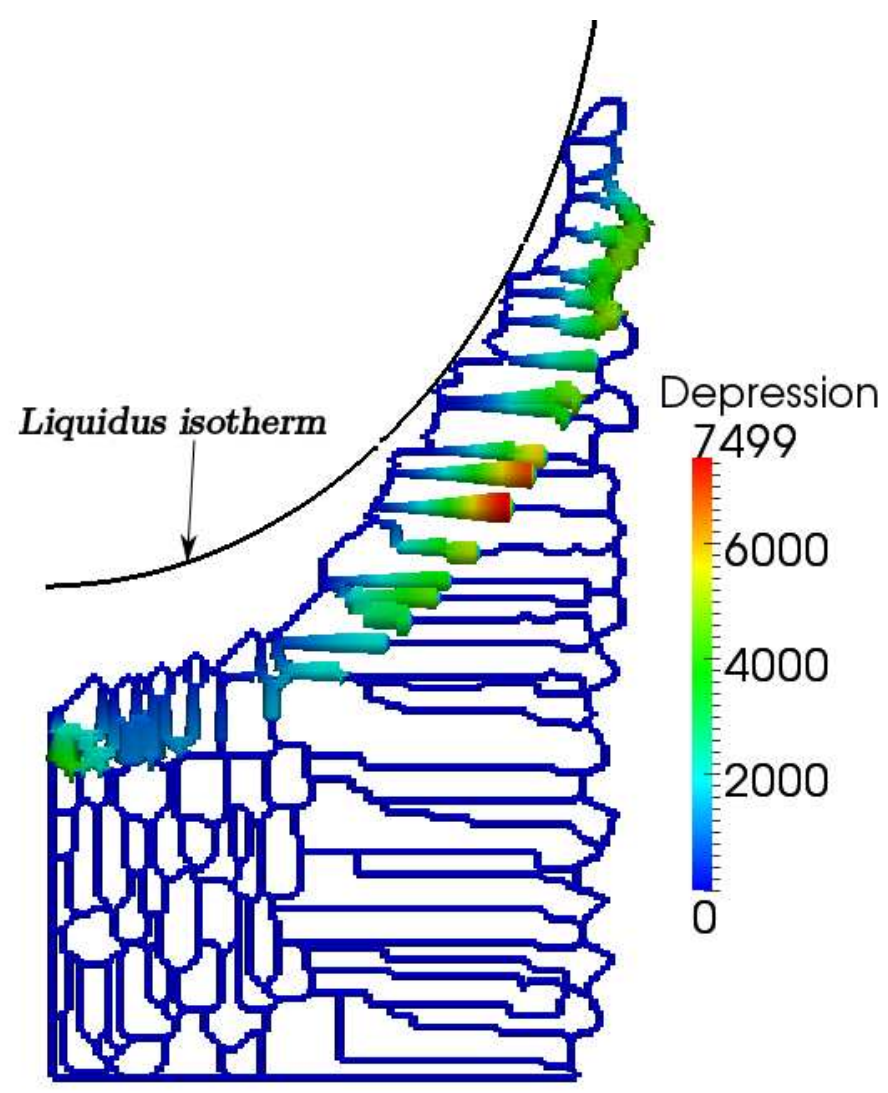

Figure 7: Pressure drop in $\mathrm{Pa}$ in the intergranular network (coherent solid fraction set to 0.92). Other values in the model are given in table 4

and lead to higher pressure drop.

Comparison of figures 7 and 8 indicates a maximum pressure drop with the coherent solid fraction fixed at 0.96 . The maximum pressure drop is not located at the same location due to grain boundaries activated or not in the model. By increasing the coherent solid fraction, the permeability is also decreased in equation 6 and plastic strain rate become higher and has an important effect on the strain localization phenomena.

\subsection{Strain rate localization and solidification shrinkage pressure}

To investigate strain rate localization influence on depression, the right hand side of equation 6 can be decomposed between a solidification shrinkage pressure $\Delta p_{s h}$ and a strain rate (localization) pressure drop $\Delta p_{\varepsilon}$. Because the modeling leads to a linear 
system, the total pressure drop can be decomposed as:

$$
\Delta p=\Delta p_{s h}+\Delta p_{\varepsilon}
$$

The pressure drop due to strain localization is mainly due to plastic strain rate. In equation 6 , the right hand source term associated to $\Delta p_{\varepsilon}$ is proportional to solid fraction and perpendicular plastic strain rate. In order to see the distribution of plastic strain rate perpendicular to the grain boundary is shown in figure 10 .

The highest plastic strain rate is seen in the equiaxed grains region. It means that at constant solid fraction, the critical site due to strain localization will be in the equiaxed zone. The problem is a little bit more complicated due to the non homogeneous fields and due to structural effects. The pressure drop is the result of high strain rate localization and to the incapacity of the grain boundaries to let the metal flow and feed the grain boundary opening due to the strain localization. In other words, if there is a strong strain localization but that the fluid network can feed the opening between two grains. The fluid flow compensate teh opening and no pore nucleates.

To appreciate the influence of strain localization to pressure drop the same simulation as the one of figure 7 is performed but with forcing the pressure drop due to shrinkage equal to zero. The result is shown in figure 10 .

The highest pressure drop is located in columnar zone and it is not located where there is the highest strain rate.

\section{Discussion}

The pressure drop is influenced by the solidification shrinkage and by the plastic strain rate as well as the capacity of the fluid to flow along grain boundaries. The model is not intend to be predictive. The modeling wants to see if the coupling between process simulation, microstructure generation and fluid flow along the grain boundaries can give information on the critical location for crack onset. In this term, the model reproduced the critical site along columnar grain boundaries. The highest depression is around $10 \mathrm{kPa}$ and is higher that depression measured in the liquid metal probably due to rapid solidification effects that are not taken into account in the model. 
It has to be outlined, that our model is nevertheless a poor representation of the morphology and of the real behaviour of the solidifying microstructure. Different points can be improved. First, The morphology of the intergranular network follows the CA grid and doesn't take into account any curvature of the grain boundaries. Secondly, the behaviour of the liquid network can be discontinuous whereas in reality the fluid network is often continuous [30]. If the media is three dimensional, the critical site can easily be fed in a continuous network. Third, the model is non representative due to the non-dependence of the solid fraction in function of species concentration due to micro or macro segregation and to the non dependence on the solidification path.

The model developed considers no flow between the grain boundaries and the adjacent grains. In some metal it can be a poor assumptions and some improvements in the model must be done by considering fluid flow inside the grain and a mass exchange between the grain boundary and the grain.

The important features of the model are the couplings between the thermal and mechanical modelling at the process scale, the microstructure prediction based on a cellular automaton and the pressure prediction inside the intergranular network. The article seems to demonstrate that this kind of analysis is necessary to improve the understanding of hot cracking in welding. The model can detect the location of the critical site in the columnar grains boundaries as observed in [5]. If it is now not predictive it can be used to optimize the process parameters to decrease the temperature rate in the critical zone by adding a heat source for example.

\section{Conclusion}

A new approach was developed in this paper that combines process simulations, microstructural prediction and multiphasic model to determine pressure at grain boundaries. The model can be enriched by refining physical solidification models. The approach demonstrates the interest to combine different physics and scales in a model to predict hot cracking. Nevertheless, it has to keep in mind that the material behaviour under solidification is three dimensional. Some improvements can be done for the thermal transfer model by developing some thermo-convective analysis in the weld pool. In aluminium alloy, hydrogen content is known to have a critical importance on the appearance of pore 
and then cracks and must be integrated in the modelling.

\section{References}

[1] Dantzig.G, Rappaz.M, "Soldification", CRC Press, 2009

[2] Chihoski.R.A, "Understanding weld cracking in aluminium sheet". Welding Journal,1972, January p24 30

[3] Wang.N, Mokadem.S, Rappaz.M, Kurz.W "Solidification cracking of superalloy single and bicrystals" Acta Materiala, 2004, Vol 52, p3173-3182

[4] Norman.A.F, Drazhner.V and Prangnell.P.B "Effect of welding parameters on the solidification microstructure of autogeneous TIG welds in an Al-Cu-Mg-Man alloy", Materials Science and Engineering A, 1999, Vol 259,p53-64

[5] Niel.A, Bordreuil.C, Deschaux-Beaume.F and Fras.G "Modeling hot cracking phenomena in 6061 Aluminum alloy weld with a microstructural based criteria", Science and Technology of Welding and Joining, 2013, http://dx.doi.org/10.1179/1362171812Y.0000000072

[6] Hot cracking phenomena in welds, Ed. Bollinghaus and Herold, Springer,2005

[7] Hot cracking phenomena in welds, Ed. Bollinghaus and Herold and Cross and Lippold, Springer,2008

[8] Hot cracking phenomena in welds, Ed. Lippold and Bollinghaus and Cross, Springer,2011

[9] F. Matsuda, H. Nakagawa, and K. Sorada." Dynamic observation of solidification and solidification cracking during welding with optical microscope (i) - solidification front and behavior of cracking". Trans. Jap. Weld. Ins., 1982, Vol 11, p67 77

[10] Rappaz.M, Drezet.J-M and Gremaud.M "A new hot tearing criterion", Metallurgical Transactions A, 1999, Vol 30, p449-455

[11] Safari.A.R, Forouzan.M.R, Shamanian.M, "Hot cracking in stainless steel 310s, numerical study and experimental verification", Computational Materials Science, 2012, Vol 63, p182-190

[12] Rappaz.M, Gandin Ch-A, Desbiolles.J-L and Thevoz.P "Prediction of grain structures in various solidification processes" Metallurgical and materials transactions A, 1996, vol 27, p695-705

[13] Atwood.R.C and Lee.P.D "A three-phase model of hydrogen pore formation during the equiaced dendritic solidification of aluminium silicon alloys", Metallurgical and materials transactions B, 2002, Vol 33B, p209-221

[14] Coniglio.N and Cross.E, "Mechanisms for solidification crack initiation and growth in aluminum welding", Metallurgical and materials transactions A, 2009, Vol 40, p2718-2727

[15] Farzadi.A, Do-Quang.M, Serajzadeh.S, Kokabi.A and Amberg.G "Phase Field simulation of weld solidification microstructure in an $\mathrm{Al} \mathrm{Cu}$ alloy" Modeling and simulations in Materials Science and Engineering, 2008, Vol16 doi:10.1088/0965-0393/16/6/065005

[16] Niel.A, Phd thesis of Université Montpellier 2, 2011

[17] Maisonnette.D "Influences mcaniques et mtallurgiques de procds hautes tempratures sur un alliage 6061". PhD thesis, INSA Lyon, 2010.

[18] Zavanglios.A, "Modeling of the mechanical behavior of semi-solid metallic alloys at high volume fractions of solid", International journal of mechanical science, 1998, Vol 40,p1029-1041

[19] http://www.cgal.org

[20] Dress.W.B, Zacharia.T and Radhakrishnan.B, "Cellular Automata Modeling of Weld Solidification Structure", International Conference on Modeling and Control of Joining Processes, 1993

[21] Ichikawa.K, Nogami.A, Koseki.T and Fukuda.y "Modelling of solidification and grain growth in steel welds" Mathematical modeling of weld phenomena 5 congress, 2001, Seggau Castle,

[22] Gandin Ch-A and Rappaz.M "A coupled finite element-cellular automaton model for the prediction of dendritic grain structures in solidification process", Acta Metallurgica and Materiala, 1994, Vol 42, p2233-2246

[23] Kou.S, "Welding Metallurgy", Wiley, 2003

[24] Kurz.W, Giovanola.B and Trivedi, "Theory of microstructural development during rapid solidification", Acta metallurgica, 1986, Vol 34, p823-830

[25] Hunziker.O, Dye.D and Reed.R.C "On the formation of a centreline grain boundary during fusion welding", Acta materiala, vol 48, 2000, p4191-4201

[26] Rappaz.M and Jacot.A and Boettinger.W, "Last-Stage Solidification of Alloys: Theoretical Model of Dendrite-Arm and Grain Coalescence", Metallurgical and materials transactions A, 2003, Vol $34, \mathrm{p} 467-479$

[27] Vernde.S, Jarry.Y, Rappaz.M, "A granular model of equiaxed mushy zones: Formation of a coherent solid and localization of feeding", Acta Materiala, 2006, Vol 54, p 4023-4034 
[28] Rappaz.M, Farup.M and Drezet.J-M, "Study and modeling of hot tearing formation", M.Flemings Symposium, 2000.

[29] Easton.M, Davidson.C and St John.D, "Effect of alloy composition on the dendrite arm spacing of multicomponent aluminium alloys", Metallurgical and materials transactions, A, 2010, Vol 41, p1528-1538

[30] Giraud.E and Suéry.M and Coret. "Shear behavior of AA6061 aluminium in the semisolid state under isothermal and anisothermal conditions", Metallurgical and materials transactions A, 2011, Vol42, p3370-3377 


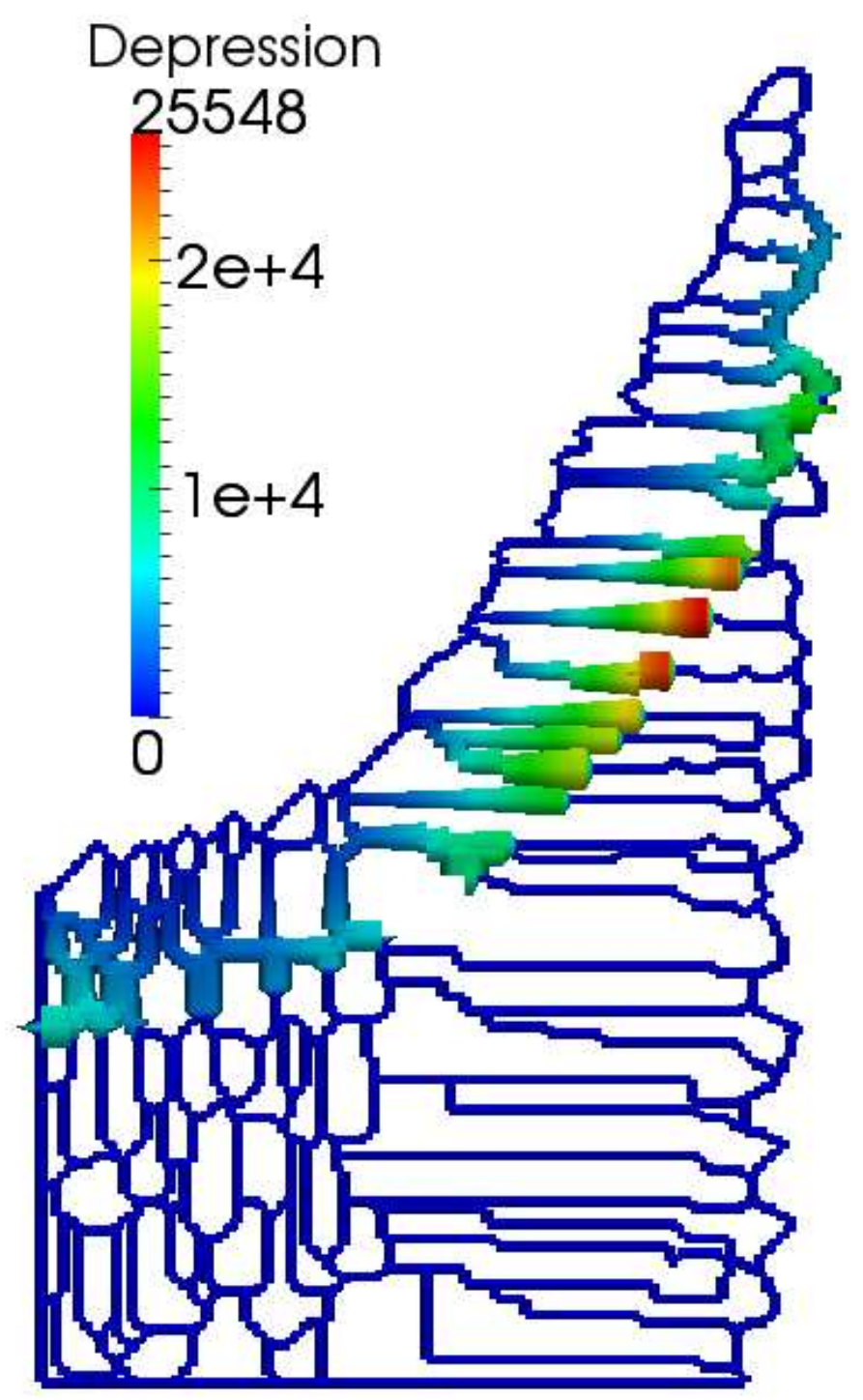

Figure 8: Pressure drop in $\mathrm{Pa}$ in the intergranular network for a coherent solid fraction set to 0.96 . Others values of the model are given in table 4. 


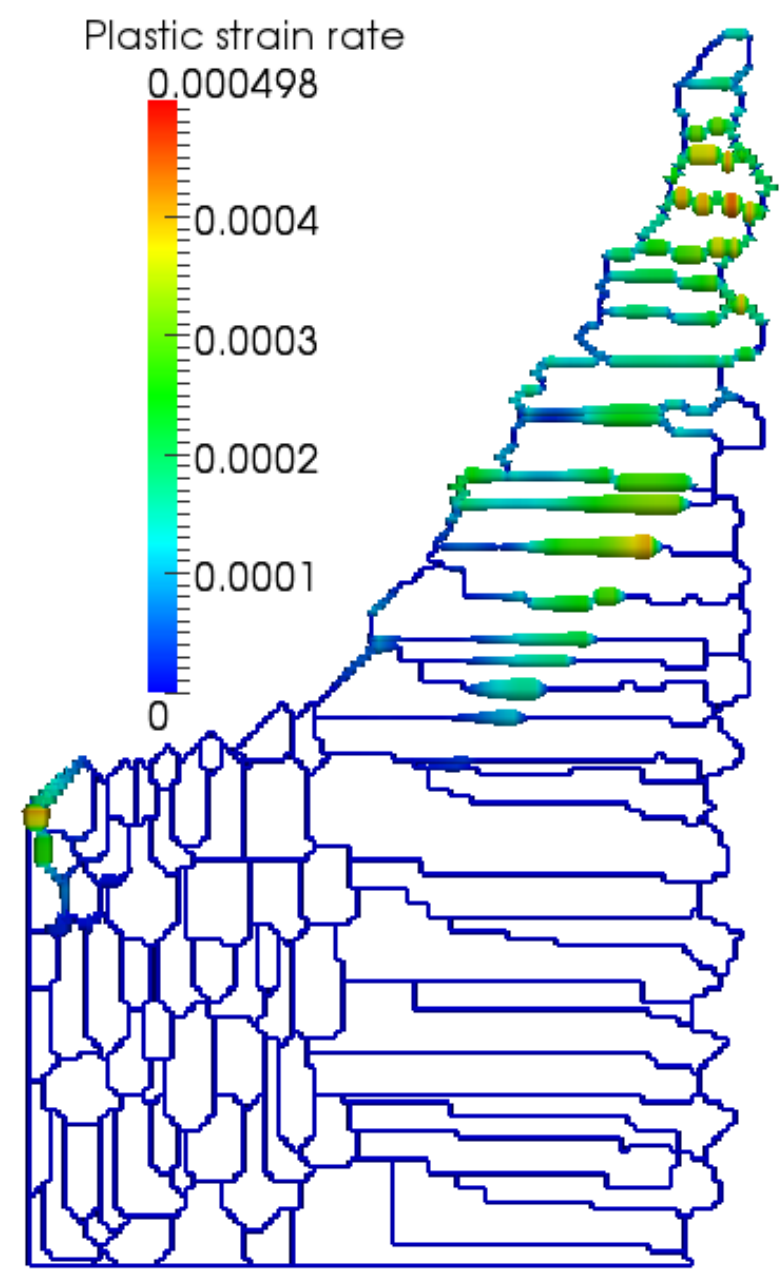

Figure 9: Perpendicular plastic strain rate distribution in the grain boundaries. Only positive strain rate are reported. 


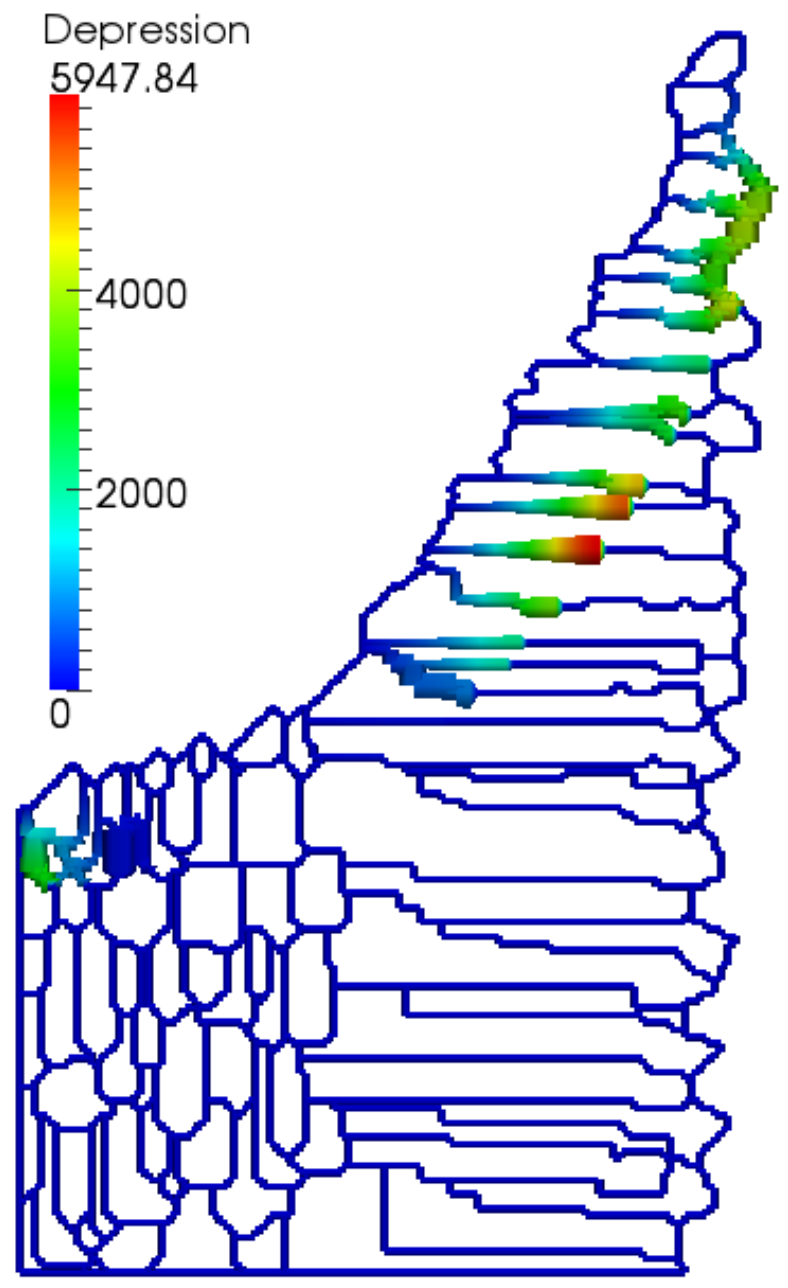

Figure 10: Pressure drop in the intergranular network due to plastic strain rate at grain boundaries for 0.92 coherent solid fraction with $K_{\text {localization }}=1000$ 